\title{
Generalizations of small profinite structures
}

\author{
Krzysztof Krupiński*
}

\begin{abstract}
We generalize the model theory of small profinite structures developed by Newelski to the case of compact metric spaces considered together with compact groups of homeomorphisms.
\end{abstract}

\section{Introduction}

In $[14,16]$ Newelski introduced the notion of a profinite structure and developed a counterpart of geometric stability theory in a purely topological setting.

A profinite structure is a pair $\left(X, A u t^{*}(X)\right)$ consisting of a profinite topological space $X$ and a closed subgroup $A u t^{*}(X)$, called the structural group of $X$, of the group of all homeomorphisms of $X$ respecting a distinguished inverse system defining $X$. We say that $\left(X, A u t^{*}(X)\right)$ is small if for every natural number $n$, there are only countably many orbits on $X^{n}$ under the action of $A u t^{*}(X)$. To develop the model theory of small profinite structures, Newelski defined $m$-independence, which has similar properties as forking independence in stable theories. He considered counterparts of such notions like Lascar $U$-rank, superstability or 1-basedness, and proved various results about them. The deepest result seems to be the group configuration theorem [16, Theorem 1.7 and Theorem 3.3].

Smallness and the fact that we have a basis consisting of clopen sets which are classes of finite $A u t^{*}(X)$-invariant equivalence relations play a prominent role in all these considerations. From the model theoretic point of view smallness is a natural assumption, because any profinite structure interpretable in a small theory (see Definition 1.2) is small. Unfortunately, it is not easy to find explicit examples of small profinite structures, especially of small profinite groups. All known examples of small profinite groups are abelian profinite groups of finite exponent and some variants of them (see $[4,5]$ for details). So it would be interesting to extend Newelski's approach to wider classes of profinite structures or even to "non-profinite" mathematical objects.

\footnotetext{
*The author was supported by a scholarship of the Foundation for Polish Science.

${ }^{0} 2000$ Mathematics Subject Classification: 03C45

${ }^{0}$ Key words and phrases: profinite structures, compact spaces, independence relation
} 
In this paper we investigate pairs $(X, G)$ where $X$ is a compact metric space and $G$ is a compact group acting continuously and faithfully on $G$ (so $G$ is just a compact subgroup of the group of all homeomorphisms of $X$ ). We call them compact structures. We assume that $(X, G)$ satisfies the existence of $m$-independent extensions (the weakest condition necessary to develop a counterpart of geometric stability theory), and we show that most of the results from [16] can be proved in this context. Notice that the class of objects that we consider contains profinite structures which are not necessarily small, but in which $m$-independent extensions exist. Sometimes we will restrict the class of compact structures to such profinite structures.

Similarly as profinite structures, compact structures appear naturally as objects interpretable in some sense in first order theories (see Definition 1.3). Namely, the space of classes of a bounded type-definable equivalence relation together with the group of homeomorphisms induced by automorphisms of the monster model is a compact structure. Moreover, any compact structure is of this form (see Theorem 1.4). We will use this fact in some proofs.

The paper is constructed as follows. In Section 1 we give definitions, prove some fundamental results about compact and profinite structures, and give a new, very short proof of Kim's theorem that in small theories the finest bounded typedefinable equivalence relation equals the relation of having the same strong type. In Section 2 we analyze the notion of $m$-independence, particularly the existence of $m$-independent extensions, and give some examples. In Section 3 we explain how to generalize some results from [16] to the case of compact structures; we also show counterparts of some results from stable (simple) theories about regular types, domination and weights (see Sections 5.1 and 5.2 of [17]).

\section{Compact and profinite structures}

In this section we give definitions and prove some fundamental results about compact and profinite structures. We also discuss some notions of interpretability of compact and profinite structures in first order theories. Finally, we give a very short proof of a theorem of Kim [3] which says that in a small theory the finest bounded $\emptyset$-typedefinable equivalence relation equals the relation of having the same strong type.

Definition 1.1 A compact structure is a pair $(X, G)$ where $X$ is a compact metric space and $G$ is a compact group acting continuously and faithfully on $X$.

Equivalently, $G$ is a compact subgroup of the group of all homeomorphisms of $X$ with compact-open topology (this topology guaranties that the action of $G$ on $X$ is continuous). Of course each profinite structure is a compact structure (recall that we always assume that the inverse system is countable).

Let $(X, G)$ be a compact structure. Let $A \subseteq X$ be finite. By $G_{A}$ we denote the pointwise stabilizer of $A$. We say that $V \subseteq X$ is $A$-invariant if $f[V]=V$ for every $f \in G_{A}$. If in addition $V$ is closed, we say that $V$ is $A$-definable. For $a \in X^{n}$ 
and $A \subseteq X$ we define $o(a / A)=\left\{f(a): f \in G_{A}\right\}$ (the orbit of $a$ over $A$ ) and $O_{n}(A)=\left\{o(a / A): a \in X^{n}\right\}$. Each orbit is always a closed subset of $X$.

For a finite $A \subseteq X$, the algebraic closure of $A$, denoted by $\operatorname{acl}(A)$, is the set of all elements in $X$ with finite orbits over $A$. If $A$ is infinite, $\operatorname{acl}(A)=\bigcup\left\{\operatorname{acl}\left(A_{0}\right)\right.$ : $A_{0} \subseteq \mathrm{A}$ is finite $\}$. We will introduce later an imaginary extension $X^{e q}$ of $X$; $a c l^{e q}$ is defined then as acl but in $X^{e q}$.

We say that compact structures $(X, G)$ and $(Y, H)$ are isomorphic, if there is a homeomorphism $\phi: X \rightarrow Y$ and an automorphism $\psi: G \rightarrow H$ such that $\phi(g x)=$ $\psi(g) \phi(x)$ for all $x \in X, g \in G$. To be precise, the definition of a profinite structure is up to isomorphism, i.e. any compact structure isomorphic to a profinite structure is also a profinite structure.

We have the following natural notion of interpretability of profinite structures in first order theories $[14,16]$ (for more details on this and another notion of interpretability see $[6])$.

Let $T$ be a first order countable complete theory $T$ with a monster model $\mathfrak{C}$, and $A \subseteq \mathfrak{C}$ be countable. In the definition below $Y$ is an arbitrary $A$-type-definable subset of $\mathfrak{C}^{e q}$ and $E_{1} \supseteq E_{2} \supseteq \ldots$ is an arbitrary descending sequence of finite $A$-definable equivalence relations on $Y$.

Definition 1.2 We say that a profinite structure is interpretable in $T$ over $A$ if it is isomorphic to the inverse limit of spaces $Y / E_{i}$ with the structural group induced by $\operatorname{Aut}(\mathfrak{C} / A)$.

So $\left(X, A u t^{*}(X)\right)$ is interpretable in $T$ over $A$ iff it is isomorphic to

$$
\left\{\left\langle a / E_{1}, a / E_{2}, \ldots\right\rangle: a \in Y\right\}
$$

with the structural group induced by $A u t(\mathfrak{C} / A)$.

The main examples of profinite structures interpretable in $T$ over $A$ are traces of complete types over $A$. More precisely, for $p \in S(A)$ we consider $\left(\operatorname{Tr}(p), A u t^{*}(\operatorname{Tr}(p))\right)$, where

$$
\operatorname{Tr}(p)=\left\{q \in S\left(a c l^{e q}(A)\right): p \subseteq q\right\}
$$

and $A u t^{*}(\operatorname{Tr}(p))$ is induced by $\operatorname{Aut}(\mathfrak{C} / A)$. We treat $\operatorname{Tr}(p)$ as the inverse limit of the system of all spaces $p(\mathfrak{C}) / E$, with $E$ ranging over finite equivalence relations on $\mathfrak{C}$ definable over $A$. So $\operatorname{Tr}(p)$ is a profinite structure homogeneous under the action of $\operatorname{Aut}^{*}(\operatorname{Tr}(p))$.

It is obvious that any profinite structure interpretable in a small theory over any finite set is small. Moreover, it is easy to show that any (small) profinite structure is interpretable as the space of all strong types in some (small) stable weakly minimal theory. To see this take any profinite structure $\left(X, A u t^{*}(X)\right)$; then we have the distinguished set $\left\{E_{i}: i \in I\right\}$ of finite invariant equivalence relations inducing the profinite topology on $X$. Let $\mathcal{X}$ be the first order structure with the universe $X$, the relations $E_{i}, i \in I$, and the relations $R_{i}, i \in I$, which are defined as follows. Write explicitly $X / E_{i}=\left\{a_{1}^{i} / E_{i}, \ldots, a_{k_{i}}^{i} / E_{i}\right\}$ and let $\pi: X \rightarrow X / E_{i}$ be the quotient 
map. Then $R_{i} \subseteq X^{k_{i}}$ is defined as $(\pi \times \ldots \times \pi)^{-1}\left[o\left(a_{1}^{i} / E_{i}, \ldots, a_{k_{i}}^{i} / E_{i}\right)\right]$, where $o\left(a_{1}^{i} / E_{i}, \ldots, a_{k_{i}}^{i} / E_{i}\right)$ is the orbit of the tuple $\left(a_{1}^{i} / E_{i}, \ldots, a_{k_{i}}^{i} / E_{i}\right)$ under the action of $A u t^{*}(X)$. Now we define $T=T h(\mathcal{X})$. Then one can check that $T$ is stable, weakly minimal and that $\left(X, A u t^{*}(X)\right)$ is interpretable in $T$ as the set of all stationary types over $\emptyset$.

For compact structures we can also introduce a natural notion of interpretability. Let $T$ be a first order countable complete theory with a monster model $\mathfrak{C}$ and $A \subseteq \mathfrak{C}$ be countable. Let $Y$ be any $A$-type-definable set and $E$ be a bounded $A$-type-definable equivalence relation on $\mathrm{Y}$. Then $Y / E$ is a compact metric space (with so called logic topology) and $A u t(\mathfrak{C} / A)$ induces a compact group (denoted by $\operatorname{Aut}(\mathfrak{C} / A)\lceil Y / E)$ acting continuously on $Y / E$ (for details see $[1,8,10]$ ).

Definition 1.3 We say that a compact structure is interpretable in $T$ over $A$ if it is isomorphic to a compact structure of the form $(Y / E$, Aut $(\mathfrak{C} / A) \uparrow Y / E)$, where $E$ is a bounded A-type-definable equivalence relation on $Y$.

Similarly as for profinite structures, it turns out that any compact structure is interpretable in some first order theory. This fact is a folklore but I have never found any published proof of it, so I give a proof below.

Theorem 1.4 Any compact structure $(X, G)$ is interpretable in some first order countable theory $T$ so that $X=\mathfrak{C} / E$ where $E$ is a bounded $\emptyset$-type-definable equivalence relation on a monster model $\mathfrak{C}$ of $T$.

Proof. Using the Haar measure on $G$ and a given metric on $X$ it is easy to produce a new metric $d$ on $X$ which is invariant under the action of $G$ (see [8], the paragraph before Theorem 3.5).

We are going to consider $X$ as a first order relational structure. Choose a dense countable subset $A$ of $X$. Let $\bar{A}$ be the set of finite tuples of elements of $A$. Now we define a countable family of relational symbols and their interpretations in $X$ :

- $U_{q}(x, y), q \in \mathbb{Q}^{+}$, and $X \models U_{q}(x, y)$ iff $d(x, y)<q$;

- $R_{a}(x), a \in \bar{A}$, and $R_{a}(X)=o(a)$.

We treat $X$ as a model in the language $\mathcal{L}=\left\{U_{q}(x, y), R_{a}: q \in \mathbb{Q}^{+}, a \in \bar{A}\right\}$. Let $T=T h(X)$ and $\mathfrak{C}$ be a monster model of $T$ containing $X$ as an elementary substructure. In fact the relations $R_{a}$ will be used only in the proof of Claim 4 below.

We define a $\emptyset$-type-definable equivalence relation on $\mathfrak{C}$ :

$$
E(x, y) \Longleftrightarrow \bigwedge_{q \in \mathbb{Q}^{+}} U_{q}(x, y)
$$

To finish the proof we need to show that $E$ is a bounded $\emptyset$-type-definable equivalence relation on $\mathfrak{C}$ and $(\mathfrak{C} / E, A u t(\mathfrak{C}) \uparrow \mathfrak{C} / E) \cong(X, G)$. We will prove this in 
successive claims.

Claim $1 \mathfrak{C} / E=\{x / E: x \in X\}$ and for any distinct $x, y \in X$ we have $x / E \neq y / E$; hence $E$ is bounded.

Proof. The second part is obvious. For the first part suppose for a contradiction that there is $a \in \mathfrak{C}$ such that $[a]_{E} \cap X=\emptyset$. Then for each $x \in X$ there is $q_{x} \in \mathbb{Q}^{+}$such that $\neg U_{q_{x}}(x, a)$. But since $X$ is compact, finitely many sets $U_{q_{x_{1}}}\left(x_{1}, X\right), \ldots, U_{q_{x_{n}}}\left(x_{n}, X\right)$ cover $X$. Hence the sets $U_{q_{x_{1}}}\left(x_{1}, \mathfrak{C}\right), \ldots, U_{q_{x_{n}}}\left(x_{n}, \mathfrak{C}\right)$ cover $\mathfrak{C}$, so $U_{q_{x_{i}}}\left(x_{i}, a\right)$ for some $i$. This is a contradiction.

Claim $2 X$ is homeomorphic to $\mathfrak{C} / E$.

Proof. Let $\pi: X \rightarrow \mathfrak{C} / E$ be the natural projection. By Claim $1 \pi$ is $1-1$ and onto. Since both spaces $X$ and $\mathfrak{C} / E$ are compact and Hausdorff, it is enough to show that $\pi$ is continuous. An open basis of the logic topology on $\mathfrak{C} / E$ consists of the sets $U_{b, q}=\left\{a / E:[a]_{E} \subseteq U_{q}(b, \mathfrak{C})\right\}, b \in X$. Then $\pi^{-1}\left[U_{b, q}\right]=U_{q}(b, X)$ is open in $X$.

From now on we identify spaces $X$ and $\mathfrak{C} / E$; then $G$ and $A u t(\mathfrak{C}) \uparrow \mathfrak{C} / E$ become compact subgroups of the group of all homeomorphisms of $X$.

Claim $3 G$ is contained in $\operatorname{Aut}(\mathfrak{C})\lceil\mathfrak{C} / E$.

Proof. It follows from the fact that $G$ consists of automorphisms of the structure $X$.

Claim 4 Aut $(\mathfrak{C})\lceil\mathfrak{C} / E$ is contained in $G$.

Proof. In the following we use compactness of $X$ and $G$, and continuity of the action. Suppose for a contradiction that there is $f \in A u t(\mathfrak{C})$ such that $f\lceil\mathfrak{C} / E \notin G$. Then there is $a=\left(a_{1}, \ldots, a_{n}\right) \in \bar{A}$ such that $\neg R_{a}\left(b_{1}, \ldots, b_{n}\right)$ where $\left\{b_{i}\right\}=\left[f\left(a_{i}\right)\right]_{E} \cap X$ for $i=1, \ldots, n$. Since $R_{a}(X)$ is closed, there is $q \in \mathbb{Q}^{+}$such that

$$
X \models\left(\forall x_{1}, \ldots, x_{n}\right)\left(\bigwedge_{1 \leq i \leq n} U_{q}\left(b_{i}, x_{i}\right) \rightarrow \neg R_{a}\left(x_{1}, \ldots, x_{n}\right)\right) .
$$

So the same formula holds in $\mathfrak{C}$, but the tuple $\left(f\left(a_{1}\right), \ldots, f\left(a_{n}\right)\right)$ witnesses that this is impossible.

One can show even more about the theory constructed above: if $(X, G)$ is homogeneous and $X$ is connected (hence $G$ is also connected), then for any strong type $p \in S_{1}\left(\operatorname{acl}^{e q}(\emptyset)\right),(X, G) \cong(p(\mathfrak{C}) / E \cap \stackrel{s}{\equiv}, A u t(\mathfrak{C} /\{p(\mathfrak{C})\})\lceil p(\mathfrak{C}) / E \cap \stackrel{s}{=})$, where $\stackrel{s}{=}$ is the relation of having the same strong type.

We see that the definition of a profinite structure depends on a distinguished inverse system. The question arises if we can define profinite structures without referring to this inverse system. The next result yields a positive answer.

Proposition 1.5 If $(X, G)$ is a compact structure such that $X$ is a profinite space, then $(X, G)$ is a profinite structure (hence $G$ is a profinite group). 
Proof. By Theorem 1.4 there exists a countable theory $T$ and a bounded $\emptyset$-typedefinable equivalence relation $E$ on a monster model $\mathfrak{C}$ of $T$ such that $(X, G) \cong$ $(\mathfrak{C} / E, \operatorname{Aut}(\mathfrak{C}) \uparrow \mathfrak{C} / E)$. Since $\mathfrak{C} / E \approx X$ is 0 -dimensional, we get that $E$ is an intersection of countably many finite $\emptyset$-definable equivalence relations $E_{i}, i \in \omega,[8$, Proposition 2.4]. We see that

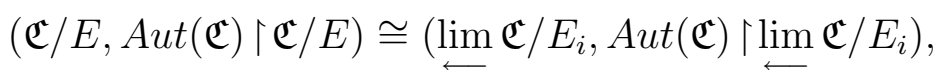

and, of course, Aut $(\mathfrak{C})$ preserves the inverse system $\mathfrak{C} / E_{i}, i \in \omega$, with the natural projections. So $(X, G)$ is a profinite structure.

Hence we can define profinite structures as those compact structures $(X, G)$ for which $X$ is a profinite space.

In model theory we can freely use names of definable sets, because we can add imaginary sorts whose elements are classes of definable equivalence relations. We can make a similar trick for compact structures.

Remark 1.6 Let $(X, G)$ be a compact structure and $E$ be a $\emptyset$-definable equivalence relation on $X^{n}$. Then $X^{n} / E$ is a compact metric space, and Aut $(X)$ induces a compact group, denoted by $\operatorname{Aut}(X) \uparrow X^{n} / E$, of homeomorphisms of $X^{n} / E$ acting continuously on $X^{n} / E$. So $\left(X^{n} / E, A u t(X)\left\lceil X^{n} / E\right)\right.$ is a compact structure.

Proof. Since $X^{n}$ is a compact metric space and $E$ is closed, we easily get that $X^{n} / E$ is a compact Hausdorff second countable space, so it is a compact metric space. The rest is an easy exercise which uses compactness of $X$ and $G$, and continuity of the action of $G$ on $X$.

Definition 1.7 Let $(X, G)$ be a compact structure. We define $X^{e q}$ as the disjoint union of sets $X^{n} / E$ with $E$ ranging over $\emptyset$-definable equivalence relations on $X^{n}$. The sets $X^{n} / E$ will be called sorts of $X^{e q}$.

By the last remark each sort of $X^{e q}$ is a compact structure. From now on our elements and sets of parameters can be taken from $X^{e q}$. As in model theory, $\left(X^{e q}\right)^{e q}=$ $X^{e q}$, which means that if $E$ is $\emptyset$-definable equivalence relation on a product of sorts $X^{n_{1}} / E_{1} \times \ldots \times X^{n_{k}} / E_{k}$, then the set of $E$-classes can be identified with the sort $X^{n_{1}} \times \ldots \times X^{n_{k}} / E^{\prime}$ where

$$
E^{\prime}\left(x_{1}, \ldots, x_{k} ; y_{1}, \ldots, y_{k}\right) \Longleftrightarrow E\left(x_{1} / E_{1}, \ldots, x_{k} / E_{k} ; y_{1} / E_{1}, \ldots, y_{k} / E_{k}\right) .
$$

Definition 1.8 Let $V$ be a definable subset of a compact structure $(X, G)$. We say that $a \in X^{e q}$ is a name for $V$ if any $f \in G$ fixes $V$ as a set iff it fixes a.

Proposition 1.9 Any set definable in a compact structure $(X, G)$ has a name in $X^{e q}$. 
Proof. Suppose $V$ is $a$-definable for some $a \in X^{e q}$. On the sort of $a$ we define an equivalence relation $E$ by:

$$
\left.E\left(a_{1}, a_{2}\right) \Longleftrightarrow\left[a_{1}=a_{2} \vee\left(a_{1}, a_{2}\right) \in S(a, a)\right)\right]
$$

where $S=\{(f, g) \in G \times G: f[V]=g[V]\}$. It is easy to check that $E$ is a $\emptyset$-definable equivalence relation and that $a / E$ is a name for $V$.

Similar definition of $X^{e q}$ was given in $[14,16]$ for profinite structures. By [14, Lemma 1.3] we know that all sorts of a small profinite structure are profinite structures. The next proposition shows that in general this is not the case.

Proposition 1.10 If $\left(X, A u t^{*}(X)\right)$ is a non-small profinite structure, then there is a $\emptyset$-definable equivalence relation $E$ on some Cartesian power $X^{n}$ such that $X^{n} / E$ is not profinite; even more, each compact metric space is of the form $X^{n} / E$ for some $E$ as above.

Proof. Replacing $X$ by $X^{n}$, if necessary, we can assume that $O_{1}(\emptyset)$ is uncountable. We know that $\left(X, A u t^{*}(X)\right)$ is interpretable as $S_{1}\left(a c l^{e q}(\emptyset)\right)$ in some first order theory $T$ (so we can identify $X$ with $\mathfrak{C} / \stackrel{s}{=}$, where $\mathfrak{C}$ is a monster model of $T$ ). Since $O_{1}(\emptyset)$ is uncountable, $S_{1}(\emptyset)$ is uncountable as well. Let $Y$ be any compact metric space. By [8, Corollary 2.3] there is a $\emptyset$-definable equivalence relation $E^{\prime}$ on $\mathfrak{C}$ coarser than the relation of having the same type and such that $\mathfrak{C} / E^{\prime} \approx Y$. Let $\pi: \mathfrak{C} / \stackrel{s}{\equiv} \rightarrow \mathfrak{C} / E^{\prime}$ be the natural projection. Define an equivalence relation $E$ on $X=\mathfrak{C} / \stackrel{s}{=}$ by

$$
E(a / \stackrel{s}{\equiv}, b / \stackrel{s}{\equiv}) \Longleftrightarrow \pi(a / \stackrel{s}{\equiv})=\pi(b / \stackrel{s}{\equiv})
$$

We see that $E$ is $\emptyset$-definable in $X$ and $X / E \approx Y$.

If $\left(X, A u t^{*}(X)\right)$ is a profinite structure, it is natural to define $X^{e q}$ as the disjoint union of those sorts $X / E$ which are profinite spaces. Then by Proposition 1.5 and Remark $1.6(X / E, \operatorname{Aut}(X) \uparrow X / E)$ is a profinite structure. It is obvious that still we have $\left(X^{e q}\right)^{e q}=X^{e q}$.

Proposition 1.11 Let $\left(X, A^{*}(X)\right)$ be a profinite space and $E$ be a $\emptyset$-definable equivalence relation. Then $X / E$ is profinite iff $E$ is an intersection of finite $\emptyset$ definable equivalence relations.

Proof. $(\Leftarrow)$ is obvious.

$(\Rightarrow)$ Since $(X / E, \operatorname{Aut}(X) \uparrow X / E)$ is a profinite structure, there is a countable family $\left\{E_{i}: i \in \omega\right\}$ of finite $\emptyset$-definable equivalence relations on $X / E$ whose classes form an open basis. Let $\pi: X \rightarrow X / E$ be the natural projection. Then $(\pi \times \pi)^{-1}\left[E_{i}\right], i \in \omega$, are finite $\emptyset$-definable equivalence relations on $X$ whose intersection equals $E$.

Proposition 1.12 Let $\left(X, A u t^{*}(X)\right)$ be a profinite structure. If $E$ is a $\emptyset$-definable equivalence relation on $X$ finer than lying in the same orbit, then $X / E$ is profinite. 
Proof. Once again we use the fact that $\left(X, A u t^{*}(X)\right)$ is interpretable as $S_{1}\left(\operatorname{acl}^{e q}(\emptyset)\right)$ in some theory $T$, and hence $X$ can be identify with $\mathfrak{C} / \stackrel{s}{=}$. Let $\pi: \mathfrak{C} \rightarrow \mathfrak{C} / \stackrel{s}{=}$ be the quotient map and $E^{\prime}=(\pi \times \pi)^{-1}[E]$. It is easy to check that $E^{\prime}$ is a $\emptyset$-type-definable equivalence relation on $\mathfrak{C}$ finer than $\equiv$ but coarser than $\stackrel{s}{=}$. By $[8$, Fact 2.5$] \mathfrak{C} / E^{\prime}$ is profinite. Since $X / E \approx \mathfrak{C} / E^{\prime}$, we are done.

The next proposition shows that $X^{e q}$ contains names for definable sets.

Corollary 1.13 Any set definable in a profinite structure $\left(X\right.$, Aut $\left.^{*}(X)\right)$ has a name in $X^{e q}$.

Proof. We see that the relation $E$ defined in the proof of Proposition 1.9 is finer than lying in the same orbit. Hence the assertion follows from Proposition 1.12.

Now we will prove some technical result which will be useful later.

Proposition 1.14 Let $(X, G)$ be a compact structure and $Z$ be an A-definable subset of $X^{e q}$ for some finite $A \subseteq X^{e q}$. Let $Y$ be a clopen subset of $Z$. Then there exists a finite $A$-definable equivalence relation $E$ on $Z$ such that $Y$ is a union of finitely many classes of $E$. In particular, the set $\left\{f[Y]: f \in G_{A}\right\}$ is finite.

Proof. Wlog $A=\emptyset$ and $X=Z$. By Theorem $1.4\left(X, A u t^{*}(X)\right)$ is interpretable in some theory $T$ as $(\mathfrak{C} / E, A u t(\mathfrak{C})\lceil\mathfrak{C} / E))$ for some bounded $\emptyset$-type-definable equivalence relation $E$. So we can identify $X$ with $\mathfrak{C} / E$. Let $E^{\prime}=E \cap \stackrel{s}{\equiv}$ and $\pi: \mathfrak{C} / E^{\prime} \rightarrow$ $\mathfrak{C} / E$ be the natural projection. We put $Y^{\prime}=\pi^{-1}[Y]$; so $Y^{\prime}$ is clopen in $\mathfrak{C} / E^{\prime}$.

Let $\tau: \mathfrak{C} / E^{\prime} \rightarrow \mathfrak{C} / \stackrel{s}{=}$ be the natural projection. By [2] or [8, Proposition 3.1] we know that pre-images of singletons by $\tau$ are connected components of $\mathfrak{C} / E^{\prime}$. Hence $\tau^{-1} \tau\left[Y^{\prime}\right]=Y^{\prime}$ and $\tau\left[Y^{\prime}\right]$ is clopen in $\mathfrak{C} / \stackrel{s}{=}$.

So there is a finite $\emptyset$-definable equivalence relation $E_{0}$ on $\mathfrak{C}$ and elements $a_{1}, \ldots, a_{n} \in$ $\mathfrak{C}$ such that $\tau\left[Y^{\prime}\right]=\sigma^{-1}\left(a_{1} / E_{0}\right) \cup \ldots \cup \sigma^{-1}\left(a_{n} / E_{0}\right)$, where $\sigma: \mathfrak{C} / \stackrel{s}{=} \rightarrow \mathfrak{C} / E_{0}$ is the natural projection.

Define an equivalence relation $E^{\prime \prime}$ on $\mathfrak{C} / E^{\prime}$ in the following way:

$$
E^{\prime \prime}\left(x / E^{\prime}, y / E^{\prime}\right) \Longleftrightarrow \sigma \tau\left(x / E^{\prime}\right)=\sigma \tau\left(y / E^{\prime}\right) .
$$

We see that $E^{\prime \prime}$ is a finite, closed and $A u t(\mathfrak{C})$-invariant equivalence relation. Moreover,

$$
Y^{\prime}=\tau^{-1} \tau\left[Y^{\prime}\right]=\tau^{-1} \sigma^{-1}\left[\left\{a_{1} / E_{0}, \ldots, a_{n} / E_{0}\right\}\right]=\left[a_{1} / E^{\prime}\right]_{E^{\prime \prime}} \cup \ldots \cup\left[a_{n} / E^{\prime}\right]_{E^{\prime \prime}} .
$$

Of course we have $\pi\left[Y^{\prime}\right]=Y$. We define a relation $F^{\prime}$ on $\mathfrak{C} / E=X$ as $(\pi \times \pi)\left[E^{\prime \prime}\right]$. The relation $F^{\prime}$ is $\emptyset$-definable in $X$, but it is not necessarily an equivalence relation. Let $F$ be the transitive closure of $F^{\prime}$. It is easy to check that $F$ is a finite $\emptyset$-definable equivalence relation on $X$ and $Y$ is a union of finitely many classes of $F$.

One can also prove the above proposition without referring to first order theories (see [7] for such a proof). 
Corollary 1.15 Let $(X, G)$ be a compact structure. Let $A, B, a \subseteq X^{e q}$ be finite and such that $o(a / A B)$ is open in $o(a / A)$. Then there exists a finite A-definable equivalence relation $E$ on $o(a / A)$ such that $o(a / A B)$ is a union of finitely many classes of $E$. In particular, the set $\left\{f[o(a / A B)]: f \in G_{A}\right\}$ is finite.

Similarly as for profinite structures we say that a compact structure is small if there are only countably many orbits over any finite set. The next remark shows that if we want to consider a class of objects essentially wider than profinite structures, we can not assume smallness.

Remark 1.16 Any small compact structure is a small profinite structure.

Proof. Suppose $(X, G)$ is a small compact structure which is not profinite. Then there is a non-trivial connected component $Y$ of $X$. Choose $y \in Y$. Then $Y$ is $y$-definable and it is covered by countably many orbits over $y$. By Baire category theorem one of these orbits is open in $Y$, but it is also closed, so it must be equal to $Y$. Hence $Y=\{y\}$, a contradiction.

The following result of Kim (see [3] or [8, Theorem 3.5]) is an immediate corollary of the last Remark and Proposition 3.1 of [8].

Theorem 1.17 In a small theory, the finest bounded $\emptyset$-type-definable equivalence relation equals $\stackrel{s}{=}$.

Proof. Let $\stackrel{b d}{\equiv}$ denote the finest bounded $\emptyset$-type-definable equivalence relation on a monster model $\mathfrak{C}$ of a small theory $T$. Then $(\mathfrak{C} / \stackrel{b d}{\equiv}, A u t(\mathfrak{C})\lceil\mathfrak{C} / \stackrel{b d}{\equiv})$ is a small compact structure. Hence, by Remark $1.16 \mathfrak{C} / \stackrel{b d}{\equiv}$ is profinite. Since by $[8$, Proposition 3.1] the strong types are connected components of $\mathfrak{C} / \stackrel{b d}{\equiv}$, the proof is completed.

\section{Properties of independence relation}

In profinite structures Newelski defined the following notion of independence relation, which plays a similar role as forking independence in stable and simple theories. Here we consider this notion in the more general context of compact structures.

Definition 2.1 Let $(X, G)$ be a compact structure, a be a finite tuple and $A, B$ finite subsets of $X$. We say that a is m-independent from $B$ over $A$ (written $a^{m} \Psi_{A} B$ ) if $o(a / A B)$ is open in o $(a / A)$. We say that a is $m$-dependent on $B$ over $A$ (written $\left.a^{m} \mathcal{L}_{A} B\right)$ if $o(a / A B)$ is nowhere dense in $o(a / A)$.

Of course if $A, B, C$ are finite subsets of $X$, then $\left.A^{m}\right\rfloor_{C} B$ means that $\left.a^{m}\right\rfloor_{C} b$ where $a, b$ are any tuples consisting of the elements of $A$ and $B$.

To develop a model theory of compact structures we need several good properties of $m^{m}$. The following was proved by Newelski [15]. 
Fact 2.1 In a small profinite structure $\left(X\right.$, Aut $\left.^{*}(X)\right)$ m-independence has the following properties.

(1) (Symmetry) For every finite $A, B, C \subseteq X$ we have that $\left.A^{m}\right\rfloor_{C} B$ iff $B^{m} \bigsqcup_{C} A$.

(2) (Transitivity) For every finite $A \subseteq B \subseteq C \subseteq X$ and $a \subseteq X$ we have that $a \mathcal{L}_{A} C$ iff $\left.a\right\rfloor^{m} \mathcal{L}_{B} C$ and $\left.a\right\rfloor^{m}{ }_{A} B$.

(3) $a \in \operatorname{acl}(A)$ implies $\left.a^{m}\right\rfloor_{A} B$ for every finite $B \subseteq X$.

(4) (Extensions) For every finite $a, A, B \subseteq X$ there is some $a^{\prime} \in o(a / A)$ with $a^{\left.\prime^{m}\right\rfloor_{A}} B$.

In fact properties (1), (2) and (3) are true for any compact structures (without smallness): (2) and (3) are trivial; (1) follows from Kuratowski-Ulam theorem applied to the subset $o(a b / C)$ of the product $o(a / C) \times o(b / C)$, where $a, b$ are any tuples of the elements of $A$ and $B$. As to Property (4), it may fail without smallness, e.g. in the additive group of $p$-addic numbers with the standard structural group or in the unit circle $S^{1}$ with the group of all rotations.

In the next section we will see that assuming Property (4), we can show most of the results proved by Newelski for small profinite structures.

Fact 2.1 is also true when we work in $X^{e q}$ instead of $X$. To show some results we have to work in $X^{e q}$.

As in the case of forking independence, from symmetry and transitivity we get

$$
\text { (*) } \left.\left.\quad a b^{m}\right\rfloor_{A} B \Longleftrightarrow a\right\rfloor_{A}^{m} B b^{m} \mathcal{L}_{A a} B
$$

for any finite $a, b, A, B \subseteq X^{e q}$.

Remark 2.2 Let $(X, G)$ be any compact structure. If Property (4) holds in $X$ lor more generally in $\left.X^{e q}\right]$ when $a$ and $B$ are singletons from $X$, then it holds in general, even for $a, B \subseteq X^{e q}$.

Proof. By transitivity and an easy induction we get that (4) holds when $a$ is a singleton and $B \subseteq X$ is finite.

Suppose now that $A, B \subseteq X$ are finite $\left[A \subseteq X^{e q}\right.$, when we work in $\left.X^{e q}\right]$. By induction on $n$ we will show that for any $a=\left(a_{1}, \ldots, a_{n}\right) \in X^{n}$ there is $a^{\prime} \in o(a / A)$ such that $a^{\prime m} \mathcal{L}_{A} B$.

Suppose that the statement holds for $(n-1)$-tuples. So there is a tuple $b=$ $\left(a_{1}^{\prime}, \ldots, a_{n-1}^{\prime}\right) \in o\left(\left(a_{1}, \ldots, a_{n-1}\right) / A\right)$ such that $b^{m} \mathcal{L}_{A} B$. Choose $a_{n}^{\prime \prime} \in X$ with $\left(a_{1}^{\prime}, \ldots, a_{n-1}^{\prime}, a_{n}^{\prime \prime}\right) \in o(a / A)$. Once again by the inductive hypothesis we get an element $a_{n}^{\prime} \in O\left(a_{n}^{\prime \prime} / A b\right)$ such that $\left.a_{n}^{\prime m}\right\rfloor_{A b} B$. So we are done by $(*)$.

Now the fact that Property (4) holds even for $a, B \subseteq X^{e q}$ easily follows from properties $(2),(3)$ and $(*)$.

Definition 2.3 We say that an orbit o $(a / A)$ in a compact structure $(X, G)$ is strongly small if for any finite $B \subseteq X$, the orbit $o(a / A)$ is a union of countably many orbits over $A B$. We say that it is small if the same condition holds but with $B \subseteq o(a / A)$. 
Remark 2.4 Each 1-orbit over $\emptyset$ is strongly small iff for every natural number $n$ each $n$-orbit over any finite subset of $X^{e q}$ is (strongly) small iff for every natural number $n$ each $n$-orbit over $\emptyset$ is small iff each orbit on any sort of $X^{e q}$ over any finite subset of $X^{e q}$ is (strongly) small.

If one of the above equivalent conditions holds, we say that $(X, G)$ has small orbits.

In the next proposition we consider a list of stronger and stronger properties between Property (4) and smallness. If $D$ is a definable set in a compact structure $(X, G)$, then $\ulcorner D\urcorner \in X^{e q}$ denotes a name of $D$.

Proposition 2.5 Let us consider the following list of properties of a compact structure $(X, G)$.

(a) Property (4) holds in $X$.

(b) Property (4) holds in $X^{e q}$.

(c) For every finite $A \subseteq X$, for every $A$-definable subset $D$ of $X$ (equivalently, of $\left.X^{e q}\right)$ such that any two elements $a, b \in D$ lie in the same orbit over $\ulcorner D\urcorner$, there is $a \in D$ such that $o(a / A)$ is open in $D$.

(d) $(X, G)$ has small orbits.

(e) For every finite $A \subseteq X$, for every $A$-definable subset $D$ of $X$ such that any two elements $a, b \in D$ lie in the same orbit over $\emptyset$, there is $a \in D$ such that o $(a / A)$ is open in $D$.

(f) For every finite $A \subseteq X$, for every $A$-definable subset $D$ of $X$, there is a $\in D$ such that $o(a / A)$ is open in $D$.

(g) $(X, G)$ is small.

Then $(a) \Longleftarrow(b) \Longleftrightarrow(c) \Longleftarrow(d) \Longleftrightarrow(e) \Longleftarrow(f) \Longleftrightarrow(g)$.

Proof. (a) $\Longleftarrow(\mathrm{b})$ is obvious.

(b) $\Longleftarrow(\mathrm{c})$. Let $a, A, B \subseteq X^{e q}$ be finite. By Remark 2.2 we can assume that $a \in X$ and $B \subseteq X$. We can identify $A$ with an element $b / E$ from some sort $X^{n} / E$. Let $D=o(a / A)$. Then $D$ is $b$-definable, so it is also $B b$-definable. Moreover, $o(a / A)=o(a / A\ulcorner D\urcorner)=o(a /\ulcorner D\urcorner)$. Hence by (c) we can find an element $a^{\prime} \in D$ such that $o\left(a^{\prime} / B b\right)$ is open in $D$. Hence $o\left(a^{\prime} / A B\right)$ is open in $o(a / A)$, i.e. $a^{\prime^{m} \downarrow}{ }_{A} B$.

$(\mathrm{b}) \Longrightarrow(\mathrm{c})$. Let $D$ satisfy the assumptions of $(c)$. We have $\ulcorner D\urcorner \in \operatorname{dcl}(A)$. Take any $a \in D$. By the assumption we have that $o(a /\ulcorner D\urcorner)=D$. Hence from (b) it follows that there is $a^{\prime} \in D$ such that $a^{\prime m^{\prime}} \downarrow_{\ulcorner D\urcorner} A$, i.e. $o\left(a^{\prime} / A\right)$ is open in D.

(c) $\Longleftarrow(\mathrm{e})$ is obvious.

(d) $\Longrightarrow$ (e) follows easily from Baire category theorem.

$(\mathrm{d}) \Longleftarrow$ (e) has a similar proof as (f) $\Longrightarrow$ (g) below. 
(e) $\Longleftarrow(\mathrm{f})$ is obvious.

(f) $\Longleftarrow$ (g) follows form Baire category theorem.

(f) $\Longrightarrow(\mathrm{g})$. We will show that there are only countably many 1-orbits over any finite set $A$. Wlog $A=\emptyset$. We construct a descending sequence $X_{\alpha}, \alpha \in \operatorname{Ord}$, of $\emptyset$-definable subsets of $X$ in the following way:

- $X_{0}=X$,

- $X_{\alpha+1}=X_{\alpha} \backslash \bigcup\left\{o(a): o(a)\right.$ is open in $\left.X_{\alpha}\right\}$,

- $X_{\gamma}=\bigcap_{\alpha<\gamma} X_{\alpha}$ for $\gamma \in \operatorname{Lim}$.

By (f) we have that if $X_{\alpha} \neq \emptyset$, then $X_{\alpha+1}$ is a proper subset of $X_{\alpha}$.

If $X_{\alpha} \neq \emptyset$ for all $\alpha<\omega_{1}$, then we get a contradiction with the fact that $X$ is second countable. Hence $X_{\alpha}=\emptyset$ for some $\alpha_{0}<\omega_{1}$. Then $X=\bigcup_{\alpha<\alpha_{0}} X_{\alpha} \backslash X_{\alpha+1}$. But since $X$ is second countable, for every $\alpha$ the set $X_{\alpha} \backslash X_{\alpha+1}$ is a union of countably many orbits which are open in $X_{\alpha}$. So we get that $X$ is a union of countably many orbits.

Below we give examples showing that (a) does not imply (b) and that (e) does not imply (f). In particular these are examples of non-small compact (profinite) structures where $m$-independent extensions exist.

As to the implication $(c) \Longrightarrow(d)$, I think it is false, but I have not found a counterexample so far.

A trivial example showing that $(e)$ does not imply $(f)$ is any uncountable compact (profinite) structure with the trivial structural group. A little bit more complicated (but still rather trivial) examples are the following.

Example 1 Let $\left(X, A u t^{*}(X)\right)$ be any small profinite structure and let $Y$ be an uncountable profinite space. Consider the profinite structure $\left(X \times Y, A u t^{*}(X)\right)$ with the trivial action of $A u t^{*}(X)$ on $Y$ and the given action of $A u t^{*}(X)$ on $X$. Then $\left(X \times Y\right.$, Aut $\left.^{*}(X)\right)$ is not small but it has small orbits, so (e) does not imply (f).

Example 1' Let $\left(X, A u t^{*}(X)\right)$ be a small profinite structure and $Y$ be a compact metric space which is not profinite. Consider the compact structure $\left(X \times Y, A u t^{*}(X)\right)$ with the trivial action of $A u t^{*}(X)$ on $Y$. Then $\left(X \times Y, A u t^{*}(X)\right)$ is a compact nonprofinite (hence non-small) structure which has small orbits.

Remark 2.6 If $(X, G)$ is a compact structure with Property (4) [in $X^{e q}$ ] and $Y$ is a compact metric space, then $(X \times Y, G)$ is a compact structure with Property (4) [in $(X \times Y)^{e q}$, respectively], where $G$ acts on $Y$ trivially.

Proof. We will show the version with imaginary sorts (the version without imaginary sorts is very easy). By Proposition 2.5 we need to show that for any $A$-definable subset $D$ of $X \times Y$ such that any two elements from $D$ lie in the same orbit over $\ulcorner D\urcorner$, there is $a \in D$ such that $o(a / A)$ is open in $D$. Let $\pi: X \times Y \rightarrow X$ be the projection on the first coordinate. Since $G$ acts trivially on $Y$, we get that $\pi[D]$ is an 
$\pi[A]$-definable subset of $X$ such that any two elements of $\pi[D]$ lie in the same orbit over $\ulcorner\pi[D]\urcorner$ (notice that $\pi$ is a bijection between $D$ and $\pi[D]$ ). So by the assumption and Proposition 2.5 we can find an $a^{\prime} \in \pi[D]$ such that $\left.o\left(a^{\prime} / \pi[A)\right]\right)$ is open in $\pi[D]$. Let $a \in D$ be such that $\pi(a)=a^{\prime}$. We see that $o(a / A)$ is open in $D$.

One can easily check that if $(X, G)$ and $(Y, H)$ are small [or with small orbits, or with Property (4)] compact structures, then so is $(X \times Y, G \times H)$ with the natural action of $G \times H$ on $X \times Y$. I do not know, however, if the same implication holds for Property (4) in imaginary sorts. Any counterexample would show that the implication $(c) \Longrightarrow(d)$ is false.

Now we describe some general construction, which will yield further examples, in particular an example showing that $(a)$ does not imply $(b)$.

Construction $(*)$ Let $\left(X, A u t^{*}(X)\right)$ be a profinite structure with a distinguished inverse system $\mathcal{X}=\left\{X_{i}, f_{j i}: i \leq j ; i, j \in I\right\}$, i.e. $X$ is the inverse limit of $\mathcal{X}$ and $A u t^{*}(X)$ respects $\mathcal{X}$. Let $(Y, G)$ be a compact structure and let $\mathcal{Y}=\left\{Y_{i}, g_{j i}: i \leq\right.$ $j ; i, j \in I\}$ be an inverse system of compact metric spaces such that $Y$ is the inverse limit of $\mathcal{Y}$ and $G$ respects $\mathcal{Y}$.

It is clear that $X \times Y$ can be considered as the inverse limit of the system $\mathcal{X} \times \mathcal{Y}:=$ $\left\{X_{i} \times Y_{i},\left(f_{j i}, g_{j i}\right): i \leq j ; i, j \in I\right\}$. Both groups $A u t^{*}(X)$ and $G^{X}$ act naturally on $X \times Y$ as groups of permutations, and $G^{X}$ is a normal subgroup of $\operatorname{Sym}(X \times Y)$. Let $H=\operatorname{Aut}^{*}(X) \ltimes G^{X}$. Finally we define $G(X, Y)$ as the subgroup of $H$ consisting of the permutations from $H$ respecting the inverse system $\mathcal{X} \times \mathcal{Y}$.

As a topological space $H$ can be considered as the space $A u t^{*}(X) \times G^{X}$ with the product topology; then $H$ is a compact Hausdorff space (but not necessarily a topological group). It is easy to see that this topology coincides with the pointwise convergence topology coming from the action of $H$ on $X \times Y$. Then $G(X, Y)$ is a closed subset of $H$, hence it is a compact Hausdorff space.

Lemma With the above topology $G(X, Y)$ is a topological group and $(X \times Y, G(X, Y))$ is a compact structure.

Proof. Let $A_{i}$ and $G_{i}$ be the groups of homeomorphisms of $X_{i}$ and $Y_{i}$ induced by $A u t^{*}(X)$ and $G$, respectively. Since $X_{i}$ is finite, $G_{i}^{X_{i}}$ acts continuously on $X_{i} \times Y_{i}$. So $A_{i} \ltimes G_{i}^{X_{i}}$ is a compact topological group acting continuously on $X_{i} \times Y_{i}$.

Let $G_{i}\left(X_{i}, Y_{i}\right)$ be the group of permutations of $X_{i} \times Y_{i}$ induced by $G(X, Y)$. If we equip $G_{i}\left(X_{i}, Y_{i}\right)$ with the pointwise convergence topology coming from the action on $X_{i} \times Y_{i}$, then the natural map from $G(X, Y)$ onto $G_{i}\left(X_{i}, Y_{i}\right)$ is continuous, so $G_{i}\left(X_{i}, Y_{i}\right)$ is compact, and hence it is a closed subset of $A_{i} \ltimes G_{i}^{X_{i}}$. Hence $G_{i}\left(X_{i}, Y_{i}\right)$ is a compact topological group acting continuously on $X_{i} \times Y_{i}$. Finally $G(X, Y)$ is the inverse limit of all these groups (so it is a compact topological group) and it acts continuously on $X \times Y$.

Now we use Construction $(*)$ to produce some examples. In all applications of Construction $(*)$ we assume that $I=\omega$, i.e. the inverse systems are indexed by 
natural numbers.

Example 2 Let $\left(X, A u t^{*}(X)\right)$ be any small profinite structure [or more generally a profinite structure satisfying Property (4)] such that $\operatorname{acl}(A)=A$ for any finite subset $A$ of $X$. Let $\left(Y, A u t^{*}(Y)\right)$ be an infinite strongly 1-transitive profinite structure, i.e. all points lie in the same orbit and if we fix any point, orbits are singletons. Then $(X \times Y, G(X, Y))$ satisfies Property (4), but it does not satisfy Property (4) in $(X \times Y)^{e q}$. This shows that $(a)$ does not imply $(b)$.

Proof. Let $A \subseteq B$ be finite subsets of $X \times Y$ and $a=\left(a_{1}, a_{2}\right) \in X \times Y$. We need to show that there is $a^{\prime} \in o(a / A)$ such that $o\left(a^{\prime} / B\right)$ is open in $o(a / A)$.

Let $A_{1}, B_{1}$ be the projections of $A$ and $B$ on the first coordinate and $A_{2}, B_{2}$ on the second one. If $a_{1} \in A_{1}$, then $o(a / A)$ is a singleton, hence $a^{\prime}=a$ does the job. If $a_{1} \notin A_{1}$, then there is an open subset $U$ of $Y$ such that $o\left(a_{1} / A_{1}\right) \times U$ is an open subset of $o(a / A)$. Now, since $\left(X, A u t^{*}(X)\right)$ satisfies Property (4), there is $a_{1}^{\prime} \in o\left(a_{1} / A_{1}\right)$ such that $o\left(a_{1}^{\prime} / B_{1}\right)$ is open in $o\left(a_{1} / A_{1}\right)$. Then $a_{1}^{\prime} \notin B_{1}$, so if we take any $a_{2}^{\prime} \in U$, we get that $o\left(\left(a_{1}^{\prime}, a_{2}^{\prime}\right) / B\right)$ is open in $o(a / A)$. So it is enough to put $a^{\prime}=\left(a_{1}^{\prime}, a_{2}^{\prime}\right)$.

Let us prove now that $(X \times Y, G(X, Y))$ does not satisfy Property (4) in $(X \times Y)^{e q}$. Choose $a_{1} \in X$ and $a_{2} \in Y$; put $a=\left(a_{1}, a_{2}\right)$. Let $U$ be a proper, canonical open neighborhood of $a_{2}$ in $Y$. Then $U$ is $a_{2}$-definable, and hence $D:=\left\{a_{1}\right\} \times U$ is $a^{-}$ definable. Moreover, any two elements of $D$ lie in the same orbit over $\ulcorner D\urcorner$. But all orbits over $a$ on $D$ are singletons. So we see that $(X \times Y, G(X, Y))$ does not satisfy (c) in Proposition 2.5 and we are done.

Remark 2.7 If $\left(X\right.$, Aut $\left.^{*}(X)\right)$ and $(Y, G)$ have small orbits, then so has $(X \times Y, G(X, Y))$.

Proof. Take any 1-orbit $o(a)$ in $X \times Y$ and any finite subset $B$ of $X \times Y$. Let $B_{1}$ and $B_{2}$ be the projections of $B$ on both coordinates and $a=\left(a_{1}, a_{2}\right)$. Then $o(a)=o\left(a_{1}\right) \times o\left(a_{2}\right)$ and for any $a^{\prime}=\left(a_{1}^{\prime}, a_{2}^{\prime}\right) \in o(a), o\left(a^{\prime} / B\right) \supseteq o\left(a_{1}^{\prime} / B_{1}\right) \times o\left(a_{2}^{\prime} / B_{2}\right)$. To finish the proof notice that by the assumption $o\left(a_{1}\right)$ and $o\left(a_{2}\right)$ are unions of countably many orbits over $B_{1}$ and $B_{2}$, respectively.

Proposition 2.8 Let $\left(X, A u t^{*}(X)\right)$ and $\left(Y, A u t^{*}(Y)\right)$ be profinite structures.

(i) If $X$ and $Y$ are small, then $(X \times Y, G(X, Y))$ is small.

(ii) If Property (4) holds in $X$ and in $Y^{e q}$, then it also holds in $(X \times Y, G(X, Y))$.

(iii) If $X$ and $Y$ have Property (4) in imaginary sorts, then so has $(X \times Y, G(X, Y))$.

Proof. (i) is easy.

(ii) Let $A \subseteq B$ be finite subsets of $X \times Y$ and $a=\left(a_{1}, a_{2}\right) \in X \times Y$. Let $A_{1}, B_{1}$ be the projections of $A$ and $B$ on the first coordinate and $A_{2}, B_{2}$ on the second one.

If $a_{1} \in A_{1}$, then $o(a / A)=\left\{a_{1}\right\} \times o\left(a_{2} / A_{2}\right)$. Since $(Y, G)$ satisfies Property (4), we can find $a_{2}^{\prime} \in o\left(a_{2} / A_{2}\right)$ such that $o\left(a_{2}^{\prime} / B_{2}\right)$ is open in $o\left(a_{2} / A_{2}\right)$. So $a^{\prime}:=\left(a_{1}, a_{2}\right)$ does the job.

If $a_{1} \notin A_{1}$, then there is a canonical open neighborhood $U$ of $a_{2}$ such that $o\left(a_{1} / A_{1}\right) \times\left(o\left(a_{2}\right) \cap U\right)$ is an open subset of $o(a / A)$. 
Since $\left(X, A u t^{*}(X)\right)$ satisfies Property (4), there is $a_{1}^{\prime} \in o\left(a_{1} / A_{1}\right)$ such that $o\left(a_{1}^{\prime} / B_{1}\right)$ is open in $o\left(a_{1} / A_{1}\right)$. Since $\left(Y, A u t^{*}(Y)\right)$ satisfies Property (4) in $Y^{e q}$, there is $a_{2}^{\prime} \in o\left(a_{2} /\ulcorner U\urcorner\right)$ such that $o\left(a_{2}^{\prime} / B_{2}\ulcorner U\urcorner\right)$ is open in $o\left(a_{2} /\ulcorner U\urcorner\right)=o\left(a_{2}\right) \cap U$. Put $a^{\prime}=\left(a_{1}^{\prime}, a_{2}^{\prime}\right)$.

We see that $a^{\prime} \in o(a / A)$. We also have $o\left(a^{\prime} / B\right) \supseteq o\left(a_{1}^{\prime} / B_{1}\right) \times o\left(a_{2}^{\prime} / B_{2}\right) \supseteq$ $o\left(a_{1}^{\prime} / B_{1}\right) \times o\left(a_{2}^{\prime} / B_{2}\ulcorner U\urcorner\right)$ and the last product is open in $o(a / A)$. Hence $o\left(a^{\prime} / B\right)$ is open in $o(a / A)$.

(iii) Let $D \subseteq X \times Y$ be $A$-definable for some finite subset $A$ of $X \times Y$ and assume that $D$ is an orbit over $\ulcorner D\urcorner$. By Proposition 2.5 it is enough to find $a \in D$ with $o(a / A)$ open in $D$.

Let $A_{1}, A_{2}$ and $D_{1}, D_{2}$ be the projections of $A$ and $D$. Then $D_{1}$ is an $A_{1}$-definable subset of $X$ whose elements lie in the same orbit over $\left\ulcorner D_{1}\right\urcorner$. Since $\left(X, A u t^{*}(X)\right)$ satisfies Property (4) in $X^{e q}$, we can find $a_{1} \in D_{1}$ such that $o\left(a_{1} / A\right)$ is open in $D_{1}$.

If $a_{1} \in A_{1}$, then $D_{1}$ is finite and it is enough to apply the assumption that $Y$ satisfies Property (4) in $Y^{e q}$ to the $A_{2}$-definable set $\pi_{2}\left[\left(\left\{a_{1}\right\} \times Y\right) \cap D\right]$, where $\pi_{2}: X \times Y \rightarrow Y$ is the projection on the second coordinate.

Assume now that $a_{1} \notin A_{1}$. Take any $a_{2} \in Y$ with $\left(a_{1}, a_{2}\right) \in D$. Put $a=$ $\left(a_{1}, a_{2}\right)$. Then we can choose a canonical open neighborhood $U$ of $a_{2}$ such that $o\left(a_{1} / A_{1}\right) \times\left(o\left(a_{2}\right) \cap U\right) \subseteq o(a / A) \subseteq D$. Of course $o\left(a_{1} / A_{1}\right) \times\left(o\left(a_{2}\right) \cap U\right)$ is open in $D_{1} \times o\left(a_{2}\right)$. On the other hand, since all elements in $D$ lie in the same orbit over $\emptyset$, we have $D \subseteq D_{1} \times o\left(a_{2}\right)$. Hence $o(a / A)$ is open in $D$.

Let us introduce the following terminology.

Definition 2.9 A compact e-structure is a compact structure satisfying Property (4), and a compact ei-structure is a compact structure satisfying Property (4) in imaginary sorts.

We already know that there exist profinite $e i$-structures which are not small and compact $e i$-structures which are not profinite. However, we have the following proposition.

Proposition 2.10 All orbits in a compact ei-structure are profinite.

Proof. Let $o$ be any orbit. We need to show that all connected components of $o$ are trivial. Let $D$ be the connected component of an element $a \in o$. Then $D$ is $a$ definable and any two elements of $D$ lie in the same orbit over $\ulcorner D\urcorner$. By Proposition 2.5 there is $b \in D$ such that $o(b / a)$ is clopen in $D$. So $D=\{a\}$.

Following Newelski, we say that a sequence $\left(a_{i}: i \in \omega\right)$ is a flat Morley sequence in an orbit $o=o(a / A)$ in a compact structure $(X, G)$ if it is $m$-independent (i.e. $o\left(a_{n} / A a_{<n}\right)$ is open in $o$ for every $\left.n\right)$ and dense in $o$.

Remark 2.11 Morley sequences exist in compact ei-structures. 
This remark follows easily from Proposition 2.10 and the fact that names of canonical open sets are algebraic over $\emptyset$. This shows even more, namely, that Morley sequences in orbits over standard sets exist in every profinite $e$-structure $\left(X, A_{u} t^{*}(X)\right)$ that satisfies Property (4) over finite subsets of $X \cup a c l^{e q}(\emptyset)$. Notice that the profinite $e$-structure constructed in Example 2 has this property but it is not a profinite $e i$-structure.

\section{Model theory of compact $e$-structures}

\subsection{Newelski's results in a wider context}

In this section we explain why most of the results (including the group configuration theorem) proved by Newelski for small profinite structures hold in the more general context of compact (profinite) $e i$-structures. In fact some basic results hold even in compact $e$-structures. We also mention some results which cannot be generalized to the case of compact $e i$-structures.

Essentially, in model theory of small profinite structures we use the following consequences of smallness:

(a) Property (4);

(b) Property (4) in imaginary sorts;

(c) Existence of open orbits in definable sets.

A general rule is that any result about small profinite structures whose proof uses only (a) [or (b)] holds for any profinite $e$-structure [ei-structure]. If a proof uses (c), then we cannot automatically generalize it to the case of profinite $e i$-structures.

Similarly, if we look at the results about small profinite structures, we see that the fact that the universe is a profinite space is used in the following way:

(i) Proposition 1.14 (or only Corollary 1.15) holds;

(ii) There is a family of $\emptyset$-definable equivalence relations whose classes form a basis of open sets.

In general, any result about profinite structures whose proof uses only (i) holds for compact structures. If a proof uses (ii), we cannot automatically generalize it to the case of compact structures.

The following theorem is a generalization of [12, Theorem 1.5]. We repeat the proof in our context.

Theorem 3.1 Let $(X, G)$ be any compact structure. Let I be a ternary relation defined on finite tuples from $X^{e q}$, invariant under $G$ and satisfying properties (1), (2), (3) and (4) listed in Fact 2.1. Then ${ }^{m} \perp$ implies $I$. 
Proof. Suppose for a contradiction that $\left.a^{m}\right\rfloor_{C} b$, but $\neg a I_{C} b$ for some finite $a, b, C \subseteq$ $X^{e q}$. Then $o(a / C b)$ is open in $o(a / C)$. By Corollary 1.15 we get that $d:=\ulcorner o(a / C b)\urcorner \in$ $\operatorname{acl}^{e q}(C)$.

Let us prove now that $\neg a I_{C d} b$. Otherwise, by (1) we get $b I_{C d} a$. By (3) we have $d I_{C} a$. So by (1) and (2) we get $b d I_{C} a$, hence $a I_{C} b d$, and finally $a I_{C} b$, a contradiction.

Since $d$ is a name for $o(a / C b)$, we have that $o(a / C d)=o(a / C d b)$. Take any $a^{\prime} \in o(a / C d)$. Then $a^{\prime} \in o(a / C d b)$. Since $I$ is invariant under $G$, we get $\neg a^{\prime} I_{C d} b$; this contradicts Property (4) for $I$.

From now on assume that $(X, G)$ is a compact (profinite) $e$-structure. Working in $X^{e q}$ we additionally assume that it is a compact $e i$-structure.

We will recall some notions and results of Newelski, but in our wider context. Instead of repeating proofs, we will briefly explain why they work, referring the reader to Newelski's papers for details.

We can measure the size of orbits in $X$ (or $\left.X^{e q}\right)$ by means of the rank function $\mathcal{M}$.

Definition 3.2 The rank $\mathcal{M}$ is the unique function from the collection of orbits over finite sets to the ordinals together with $\infty$, satisfying

$$
\begin{aligned}
& \mathcal{M}(a / A) \geq \alpha+1 \quad \text { iff } \text { there is a finite set } B \supseteq A \text { such that } \\
& \\
& o(a / B) \text { is nowhere dense in } o(a / A) \text { and } \mathcal{M}(a / B) \geq \alpha .
\end{aligned}
$$

The following results follow from a standard forking calculation (e.g. see [17, Lemma 5.1 .4 and Theorem 5.1.6]. In the next proposition $a, b, A$ are finite tuples (subsets) of $X$ or $X^{e q}$.

Proposition 3.3 1. $\left.a^{m}\right\rfloor_{A} b$ iff $\mathcal{M}(a / A b)=\mathcal{M}(a / A)$.

2. $\mathcal{M}(a / b A)+\mathcal{M}(b / A) \leq \mathcal{M}(a b / A) \leq \mathcal{M}(a / b A) \oplus \mathcal{M}(b / A)$.

3. Suppose $\mathcal{M}(a / A b)<\infty$ and $\mathcal{M}(a / A) \geq \mathcal{M}(a / A b) \oplus \alpha$. Then $\mathcal{M}(b / A) \geq$ $\mathcal{M}(b / A a)+\alpha$.

4. Suppose $\mathcal{M}(a / A b)<\infty$ and $\mathcal{M}(a / A) \geq \mathcal{M}(a / A b)+\omega^{\alpha} n$. Then $\mathcal{M}(b / A) \geq$ $\mathcal{M}(b / A a)+\omega^{\alpha} n$.

5. If $a^{m} \mathcal{L}_{A} b$, then $\mathcal{M}(a b / A)=\mathcal{M}(a / b A) \oplus \mathcal{M}(b / A)$.

As in stable or simple theories, the inequalities in the point (2) are called Lascar inequalities.

Remark 3.4 Let $a, A \subseteq X$ be finite. Then the $\mathcal{M}$-rank of o(a/A) computed in $X$ is the same as the $\mathcal{M}$-rank of o $(a / A)$ computed in $X^{e q}$.

Definition $3.5(X, G)$ is $m$-stable if every 1-orbit has an ordinal $\mathcal{M}$-rank.

Equivalently, there is no infinite sequence $A_{1} \subseteq A_{2} \subseteq \ldots$ of finite subsets of $X$ and $a \in X$ such that $o\left(a / A_{i+1}\right)$ is nowhere dense in $o\left(a / A_{i}\right)$ for every $i$.

By Lascar Inequalities we easily get: 
Remark $3.6(X, G)$ is $m$-stable iff each $n$-orbit has an ordinal $\mathcal{M}$-rank iff each $n$-orbit in $X^{e q}$ has an ordinal $\mathcal{M}$-rank.

The following fact proved by Newelski for small profinite structures [15, Lemma 2.6] also holds in our context (since its proof uses only properties (b) and (i)).

Proposition 3.7 Assume that $(X, G)$ is m-stable and $a, A \subseteq X^{e q}$. Then $o(a / A)$ is invariant over finitely many parameters from o $(a / A)$.

The next definition (see [16]) is in the same spirit as the above result; in fact it is a counterpart of the notion of 1-basedness in geometric stability theory. However, in compact structures we have to formulate it in a little bit different way than in [16] (both definitions coincide in profinite structures).

Definition $3.8(X, G)$ is m-normal if for every finite $a, A \subseteq X$, there is $U \ni a$ clopen in $o(a / A)$ and with finitely many conjugates under $G_{a}$.

The next two results are more or less Theorem 2.3 of [15]. However, we will prove them in our context to ilustrate how Proposition 1.14 works.

Remark $3.9(X, G)$ is m-normal iff for every finite $a, A \subseteq X^{e q}$, there is $U \ni$ a clopen in $o(a / A)$ and with finitely many conjugates under $G_{a}$.

Proof. $(\Longleftarrow)$ is obvious.

$(\Longrightarrow)$. Take any $a, A \in X^{e q}$. Then $A=A_{0} / F$ where $A_{0}$ is a finite tuple from $X$ and $F$ is a $\emptyset$-definable equivalence relation. Wlog $A_{0}{ }^{m} \mathcal{L}_{A} a$, hence $a^{m} \mathcal{L}_{A} A_{0}$. This means that $o\left(a / A_{0}\right)$ is clopen in $o(a / A)$. Hence, wlog $A=A_{0}$.

Now, $a=a_{0} / E$ for some finite tuple $a_{0}$ from $X$ and a $\emptyset$-definable equivalence relation $E$. Wlog

$$
\text { (*) } \quad a_{0}{ }^{m} \downarrow_{a} A .
$$

Since $(X, G)$ is $m$-normal, we can find a set $V \ni a_{0}$ clopen in $o\left(a_{0} / A\right)$ and with finitely many conjugates under $G_{a_{0}}$.

Let $b=\ulcorner V\urcorner$. Then $b \in a c^{e q}\left(a_{0}\right)$. On the other hand, by Proposition 1.14 $b \in \operatorname{acl}^{e q}(A)$. This together with $(*)$ gives us

$$
(* *) \quad b \in \operatorname{acl}^{e q}(a) .
$$

Moreover, by Proposition 1.14 we can assume that $V$ is the equivalence class $\left[a_{0}\right]_{E_{0}}$ of some finite $A$-definable equivalence relation $E_{0}$ on $o\left(a_{0} / A\right)$. Indeed, by Proposition 1.14 there is a finite $A$-definable equivalence relation $E_{0}$ on $o\left(a_{0} / A\right)$ such that $\left[a_{0}\right]_{E_{0}} \subseteq$ $V$. Since $\left[a_{0}\right]_{E_{0}}$ is clopen in $V, V$ is $b$-invariant and $b \in \operatorname{acl}^{e q}\left(a_{0}\right)$, we get that $\left\{f\left[\left[a_{0}\right]_{E_{0}}\right]: G_{a_{0}}\right\}$ is finite. Hence wlog $V=\left[a_{0}\right]_{E_{0}}$.

Let $T: X \rightarrow X / E$ be the quotient map.

Claim $T[V]$ is a clopen subset of $o(a / A)$.

Proof. $G_{A b}$ acts transitively on $V$, so it acts transitively on $T[V]$. On the other hand, 
since $o\left(a_{0} / A\right)$ is covered by finitely many translates of $V$ by elements of $G_{A}, o(a / A)$ is also covered by the translates of $T[V]$ by these same elements of $G_{A}$. Hence $T[V]$ has a nonempty interior in $o(a / A)$, so it is open by the first line of the proof. The fact that $T[V]$ is closed follows from continuity of $T$ and compactness of $X$.

Claim tells us that $T[V]$ is a clopen neighborhood of $a$ inside $o(a / A)$. By $(* *)$ we get that $T[V]$ has finitely many conjugates under $G_{a}$.

The next result shows that $m$-normality corresponds to the notion of 1 -basedness.

Proposition $3.10(X, G)$ is m-normal iff for all finite $a, b \subseteq X^{e q}$, there exists $c \in$ $a c l^{e q}(a) \cap a c l^{e q}(b)$ with $a^{m} \Psi_{c} b$.

Proof. $(\Longrightarrow)$ Take $a, b \in X^{e q}$. Then we can find a set $U \ni a$ clopen in $o(a / b)$ and with finitely many conjugates under $G_{a}$. Let $a^{+}=\ulcorner U\urcorner$. By Proposition 1.14 we get $a^{+} \in \operatorname{acl}^{e q}(a) \cap a c l^{e q}(b)$ and, moreover, wlog we can assume that $U=[a]_{E}$ for some finite $b$-definable equivalence relation $E$ on $o(a / b)$. Hence we see that $U=o\left(a / a^{+} b\right)=o\left(a / a^{+}\right)$, so $\left.a^{m}\right\rfloor_{a^{+}} b$.

$(\Longleftarrow)$ Take any finite $a, A \subseteq X$. Let $a^{+}=\ulcorner o(a / A)\urcorner$. We have $o(a / A)=o\left(a / a^{+}\right)$. By assumption there is $c \in \operatorname{acl}^{e q}(a) \cap \operatorname{acl}\left(a^{+}\right)$such that $a^{m} \Psi_{c} a^{+}$.

So $o\left(a / a^{+} c\right)$ is clopen in both $o(a / c)$ and $o\left(a / a^{+}\right)=o(a / A)$. Put $U=o\left(a / a^{+} c\right)$. Then $U$ is a clopen neighborhood of $a$ in $o(a / A)$. By Proposition 1.14 it has finitely many conjugates under $G_{c}$. Since $c \in a c l^{e q}(a), U$ has finitely many conjugates over $a$.

The next remark (see [16, Remark 1.4]) follows from Proposition 2.10.

Remark 3.11 Assume $(X, G)$ is a compact ei-structure and a, $A$ are finite subsets of $X^{e q}$. Then $a c l^{e q}(A)=d c l^{e q}\left(A \cup a c l^{e q}(\emptyset)\right)$.

So far we have discussed some basic results of Newelski which can be generalized to compact $e i$-structures. Now we turn to some deeper results.

Lemma 3.1 of [16] is true in our context. Namely:

Proposition 3.12 Let $(X, G)$ be a compact ei-structure and o an orbit over $\emptyset$. Then $\operatorname{acl}^{e q}(a) \cap o$ is finite for every $a \in o$.

The proof of Lemma 3.1 of [16] works here, except for one step that will be explained now. As in [16], we define $Y_{a}$ as the topological closure of $a c^{e q}(a) \cap o$ and we prove that $Y_{a}=Y_{b}$ for $b \in Y_{a}$. This means that $Y_{a}$ is $a$-definable and any two elements of $Y_{a}$ lie in the same orbit over $\left\ulcorner Y_{a}\right\urcorner$. Hence by Proposition 2.5 there is an orbit over $a$ which is open in $Y_{a}$. This orbit meets $a c l^{e q}(a) \cap o$, so it is finite. Hence there is an element $b \in Y_{a}$ which is isolated in $o$. We finish in the same way as in [16]. 
Recall that $A=\{a, b, c\}$ is a dcl-triangle in $(X, G)$ if for every $x \in A$ we have $x \in \operatorname{dcl}(A \backslash\{x\}) \backslash \operatorname{acl}(\emptyset)$ and every two elements of $A$ are $m$-independent. Replacing $d c l$ by acl we get the definition of an acl-triangle.

Having the last two results and using Proposition 1.14 or 2.10 in appropriate places, we can repeat the proof of Theorem 3.3 of [16]. So we have the following group configuration theorem for compact $e i$-structures.

Theorem 3.13 Assume $\{a, b, c\}$ is an acl-triangle in an m-normal compact eistructure $(X, G)$. Then there is a group $G$, which is open in o(a), a is the neutral element of $G$ and $G$ is definable over $a$ and finitely many parameters from acleq $(\emptyset)$.

If we want to have such a theorem in the wider class of compact (profinite) $e$ structures, we have to formulate it in a little bit weaker form. The reason is that in this situation we do not have Proposition 3.12, and we do not know whether Remark 3.11 is true for compact $e$-structures.

Definition 3.14 A compact structure $(Y, H)$ is interpretable in $(X, G)$ over a finite subset $A$ of $X$ if there is a closed subgroup $H^{*}$ of $H$ and $A$-definable subset $Z$ of $X^{e q}$ such that $\left(Y, H^{*}\right)$ is isomorphic to $\left(Z, G_{A}\right)$.

Theorem 3.15 1. Assume $\{a, b, c\}$ is an acl-triangle in an m-normal profinite $e$ structure $\left(X, A_{u t}^{*}(X)\right)$ with Property (4) over finite sets from $X \cup$ acl $^{e q}(\emptyset)$. Then there is a profinite group $\left(H, A^{*}(H)\right)$ interpretable in $\left(X, A u t^{*}(X)\right)$ over a and finitely many parameters from acl ${ }^{e q}(\emptyset)$.

2. Assume $\{a, b, c\}$ is a dcl-triangle in an m-normal compact e-structure $(X, G)$ with Property (4) over finite sets from $X \cup a c l^{e q}(\emptyset)$. Then there is a compact group $(H, K)$ interpretable in $(X, G)$ over a and finitely many parameters from acl ${ }^{e q}(\emptyset)$.

To prove this theorem it is enough to apply Newelski's proof of Theorem 3.3 of [16], using Proposition 1.14 in appropriate places. The group $H$ obtained in Newelski's proof together with $K$ defined as the pointwise stabilizer of $a$ and finitely many parameters from $a c l^{e q}(\emptyset)$ named in the proof do the job.

There are many other results which can be generalized to the case of compact (profinite) $e i$-structures. We will briefly discuss some of them.

In $[12,13,15,16]$ Newelski considered acl-pregeometry on an orbit of $\mathcal{M}$-rank 1. To have some good properties (e.g. homogeneity) of this pregeometry we need to localize it at a flat Morley sequence. Since we do not know if flat Morley sequences exist in compact (profinite) $e$-structures, we should rather concentrate on compact $e i$-structures (where flat Morley sequences always exist). Newelski introduced the notion of full (weak) coordinatization and he proved [15, Theorem 3.3] that a small profinite structure of finite $\mathcal{M}$-rank is $m$-normal iff it has full (weak) coordinatization and each orbit of $\mathcal{M}$-rank 1 is locally modular. Analyzing Newelski's proof, and modifying it appropriately, we can conclude that this equivalence is also true for compact $e i$-structures (the only problem is that we need to apply Theorem 1.4 of 
[11], whose proof uses the existence of open orbits in definable sets; fortunately, we can omit this obstacle using Proposition 2.5).

Similarly as in [16], we get the following corollary of Theorem 3.13, which is another form of the group configuration theorem.

Corollary 3.16 If $(X, G)$ is a compact ei-structure with a non-trivial locally modular orbit o, of $\mathcal{M}$-rank 1 , then some open subset of of o is a definable group.

In my opinion the group configuration theorem is the deepest result concerning small profinite structures. The fact that this result (and many others) holds for compact $e i$-structures shows that the existence of $m$-independent extensions is a sufficient assumption.

On the other hand, there are some results $[14,18,9]$ which describe the structure of small profinite groups and rings, and which significantly restrict the class of possible examples of small profinite groups (recall that all known examples of small profinite groups are some variants of abelian profinite groups of finite exponent $[4,5]$ ). Most of these results are based on various chain conditions proved in Section 2 of [14], and on the fact that small profinite groups are locally finite [14, Proposition 2.4]. In compact (profinite) ei-groups these results are false (to see this consider infinite profinite groups with the trivial structural group). So there is a chance to get some interesting examples of compact (profinite) ei-groups. I do not know, for example, whether the free profinite group on infinitely many generators with some natural (standard) structural group satisfies the existence of $m$-independent extensions (whereas we know that it is not small, since it is not locally finite).

\subsection{Regular orbits, domination and weights}

In this section we study counterparts of some model theoretic notions (which have not been considered in the context of small profinite structures so far) in our general context of compact $e i$-structures. The main result of this section is Theorem 3.24 saying that each orbit in an $m$-stable compact $e i$-structure is equidominant with a product of finitely many regular orbits.

From now on $(X, G)$ is an $m$-stable compact $e i$-structure. The general scheme is the same as in Sections 5.1 and 5.2 of [17]. However, caution has to be taken because we do not have stationary (Lascar strong) types, independence theorem and canonical bases. The following, stronger version of Proposition 3.7 will allow us to omit all such obsatcles.

Proposition 3.17 Let $a, A, B \subseteq X^{e q}$ be finite. Then in $o(a / A)$ there exists a finite sequence $\left(a_{i}\right)_{i \leq n} m$-independent over $A, m$-independent from $B$ over $A$, and such that o(a/A) is invariant over $\left\{a_{i}: i \leq n\right\}$.

Proof. Wlog $a^{m} \mathcal{L}_{A} B$ and $A \subseteq B$. Let $b=\ulcorner o(a / A)\urcorner$. We use induction on $\mathcal{M}(b)$.

Case 1. $\mathcal{M}(b)=0$. This means that $b \in a c l^{e q}(\emptyset)$ and hence $X_{0}:=o(a / A)$ has finitely many conjugates $X_{0}, \ldots, X_{n}$ under $G$. This implies that $X_{0}$ is not a subset 
of $X_{i}$ for every $i \neq 0$. Now we see that for any $a_{i} \in X_{0} \backslash X_{i}$, the orbit $o(a / A)$ is invariant over $\left\{a_{i}: i \leq n\right\}$.

So we need to prove that we can find $a_{i} \in X_{0} \backslash X_{i}, i \leq n, m$-independent over $A$ and $m$-independent from $B$ over $A$. It is enough to show that for any finite $C \subseteq X^{e q}$ and for every $i \leq n$ there is $a^{\prime} \in X_{0} \backslash X_{i}$ with $a^{\prime^{m} \downarrow}{ }_{A} C$.

Since $X_{0} \backslash X_{i}$ is clopen in $o(a / A)$, by Proposition 1.14 we get that $c:=\left\ulcorner X_{0} \backslash X_{i}\right\urcorner \in$ $a c l^{e q}(A)$. We also know that $X_{0} \backslash X_{i} \neq \emptyset$, so we can find $a^{\prime} \in X_{0} \backslash X_{i}$ such that $a^{\prime m^{\prime}}{ }_{A c} C$. Then $a^{\prime m^{m}}{ }_{A} C$.

Case 2. $\mathcal{M}(b)=\alpha>0$. First notice that by Proposition 1.14 we get that $a^{m} \mathbb{X} A$. Since $o(a / A)=o(a / b)$, we get that $a^{m} \mathcal{X} b$, and so $b^{m}\lfloor a$. Hence

$$
(*) \quad \mathcal{M}(b / a)<\alpha .
$$

Now take $a^{\prime} \in o(a / b)$ such that $a^{\prime^{m} \bigsqcup_{b}} a$. Let $b^{\prime}$ be a name for $o\left(a^{\prime} / a b\right)$. Since $o\left(a^{\prime} / a b\right)$ is open in $o(a / b)$, by Corollary 1.15 we get that $b^{\prime} \in a c l^{e q}(b)$. So by $(*)$ and Lascar inequalities we get that $\mathcal{M}\left(b^{\prime} / a\right)<\alpha$.

Take any finite $C \supseteq B$ such that $\left.a^{m}\right\rfloor_{A} C$. Now we can use the inductive hypothesis for $o\left(a^{\prime} / a b\right)$ in $\left(X, G_{a}\right)$ to get a sequence $\left(a_{i}\right)_{i \leq k} \subseteq o\left(a^{\prime} / a b\right) m$-independent over $a b$, $m$-independent from $C$ over $a b$ and such that $b^{\prime} \in d c l^{e q}\left(a, a_{0}, \ldots, a_{k}\right)$. Since $\left.a^{\prime^{\prime}}\right\rfloor_{b} a$, we get that $a, a_{0}, \ldots, a_{k}$ are $m$-independent over $b$, and since $a^{m} \mathcal{L}_{b} C$, we get that the sequence $\left(a, a_{0}, \ldots, a_{k}\right)$ is $m$-independent from $C$ over $b$. Since $A \subseteq C$ and $b \in \operatorname{acl}^{e q}(A)$, the same is true over $A$ instead of over $b$.

We know that there are only finitely many conjugates of $o\left(a^{\prime} / a b\right): X_{0}:=o\left(a^{\prime} / a b\right)=$ $\left.f_{0}\left[o\left(a^{\prime} / a b\right)\right], X_{1}:=o\left(f_{1}\left(a^{\prime}\right) / f_{1}(a) b\right)\right)=f_{1}\left[o\left(a^{\prime} / a b\right)\right], \ldots, X_{m}:=o\left(f_{m}\left(a^{\prime}\right) / f_{m}(a) b\right)=$ $f_{m}\left[o\left(a^{\prime} / a b\right)\right]$, under $G_{b}$, and that they cover $o(a / b)$. Consider $j \leq m$. Take any finite $C \supseteq B$. We can assume that $f_{j}(a)^{m} \mathcal{L}_{b} C$. Indeed, since a name $b_{j}^{\prime}$ of $X_{j}$ belongs to $a c l^{e q}(b)$, there is $(\alpha, \beta) \in o\left(f_{j}\left(a^{\prime}\right) f_{j}(a) / b b_{j}^{\prime}\right)$ with $\alpha \beta^{m} \Psi_{b} C$; then it is enough to replace $f_{j}\left(a^{\prime}\right)$ and $f_{j}(a)$ by $\alpha$ and $\beta$, respectively. Having this, we can repeat the reasoning of the previous paragraph for $X_{j}$ instead of $X_{0}$.

So by an easy induction on $j=0,1 \ldots, m$, we get a finite sequence $\left(a_{i}\right)_{i<n}$ in $o(a / A) m$-independent over $A, m$-independent from $B$ over $A$, and such that $o(a / A)$ is invariant over this sequence.

Recall that two orbits $o(a / A)$ and $o(b / B)$ are said to be $m$-orthogonal if for any finite $C \supseteq A \cup B$ and any $a^{\prime} \in o(a / A)$ and $b^{\prime} \in o(b / A)$ with $\left.a^{\prime^{\prime}}\right\rfloor_{A} C$ and $b^{m^{m}}{ }_{B} C$ we have $a^{\prime m} \Psi_{C} b^{\prime}$. As in stable theories, we say that $o(a / A)$ is regular if it is $m$-orthogonal to all its $m$-dependent extensions.

It turns out that, as in stable theories, regular orbits are exactly the orbits on which $m$-dependence induces a pregeometry. More precisely, let $o(a / A)$ be any orbit and take any finite $B \subseteq o(a / A)$; define $\operatorname{cl}(B):=\left\{b \in o(a / A): b^{m} \mathcal{L}_{A} B\right\}$; if $B$ is infinite, put $\operatorname{cl}(B)=\bigcup\left\{c l\left(B_{0}\right): B_{0}\right.$ is a finite subset of $\left.B\right\}$.

Proposition 3.18 Assume that $a \notin a c l^{e q}(A)$. Then o $(a / A)$ is regular iff $(o(a / A), c l)$ is a pregeometry. 
Proof. $(\Longrightarrow)$. Only $\operatorname{cl}(\operatorname{cl}(B))=\operatorname{cl}(B)$ requires a proof; this can be shown by an easy forking calculus (exactly as in stable theories) and it does not use $m$-stability.

$(\Longleftarrow)$ This is a place where Proposition 3.17 or 3.7 (and hence $m$-stability) plays an important role. Suppose for a contradiction that $o(a / A)$ is regular, $\left.a^{m}\right\rfloor_{A} C, b \in$ $o(a / A), b^{m} \chi_{A} C$ and $a^{m} \chi_{C} b$.

By Proposition 3.17 we can find a finite sequence $I \subseteq o(a b / C)$ over which $o(a b / C)$ is invariant and with $\left.I^{m}\right\rfloor_{C} a b$. Hence $o(a b / I C)$ is open in $o(a b / C)$ and $o(a b / I) \subseteq$ $o(a b / C)$. So $\left.a b^{m}\right\rfloor_{I} C$.

A simple forking calculus yields: $a^{m} \Psi_{A} I, b^{m} \mathcal{X}_{A} I$ and $a^{m} \mathcal{X}_{I A} b$. This means that $a \notin \operatorname{cl}(I), b \in \operatorname{cl}(I)$ and $a \in \operatorname{cl}(I b)$, a contradiction.

The above proposition is also true for $m$-normal compact $e i$-structures. The proof is the same as the above one, except for the fact that instead of Proposition 3.17 we use the fact that for each orbit $o(a / A)$ in any $m$-normal compact $e i$-structure we can find $b \in a c l^{e q}(\emptyset)$ such that $o(a / A b)$ is invariant over $a b$. For the proof of the last fact see [11, Remark 0.2].

The proof of Proposition 5.1.11 of [17] works in our context and so we get:

Proposition 3.19 Each non-algebraic orbit is non-orthogonal to a regular orbit (of an element from $X)$.

Definition 3.20 The weight, denoted by $w$, is the unique function from the collection of all orbits over finite sets to $\omega \cup\{\infty\}$ such that for every $n \in \omega$ we have $w(o(a / A)) \geq$ $n$ iff there is a finite set $A^{\prime} \supseteq A$ and $a^{\prime} \in o(a / A)$ with $\left.a^{\prime^{\prime}}\right\rfloor_{A} A^{\prime}$, and a sequence $\left(a_{i}: i<n\right)$ independent over $\bar{A}^{\prime}$ and such that $a^{\prime m} \mathcal{X}_{A^{\prime}} a_{i}$ for every $i<n$.

The proof of Theorem 5.2.5 of [17] works in our context so we get that every orbit in any $m$-stable compact $e i$-structure has a finite weight.

The following properties of weights can be proved as in stable or simple theories $[17]$.

Proposition 3.21 1. If $a^{m} \mathcal{L}_{A} B$, then $w(a / A)=w(a / B)$.

2. $w(a b / A) \leq w(a / A)+w(b / A a)$.

3. If $a\rfloor_{A}^{m} b$, then $w(a b / A)=w(a / A)+w(b / A)$.

The next proposition is a counterpart of the well-known result for stable theories, but to prove it we use Proposition 3.17 instead of canonical bases and Morley sequences.

Proposition 3.22 Each regular orbit o $(a / A)$ has weight 1.

Proof. Suppose for a contradiction that there are $A^{\prime} \supseteq A, a^{\prime} \in o(a / A)$ with $a^{\prime^{m} \mathcal{L}_{A}} A^{\prime}$, and elements $b, c$ with $b^{m} \Psi_{A^{\prime}} c, a^{\prime^{m} \Psi_{A^{\prime}}} b$ and $a^{m^{m} \Psi_{A^{\prime}}}$. By Proposition 3.17 we can find a finite sequence $\left(b_{i}: i \leq n\right) \subseteq o\left(a^{\prime} / A^{\prime} b\right)$ which is $m$-independent over $A^{\prime} b, m$ independent from $c$ over $A^{\prime} b$, and over which $o\left(a^{\prime} / A^{\prime} b\right)$ is invariant. So there is $k \leq n$ 
such that $a^{\prime^{m}}{ }_{A^{\prime}} b_{<k}$ and $a^{\prime^{m}} \bigcup_{A^{\prime} b_{<k}} b_{k}$. The rest of the proof is the same as in the proof of Lemma 5.2.11(1) in [17]. By regularity of $o\left(a / A^{\prime}\right)=o\left(b_{k} / A^{\prime}\right)$ we have $b_{k}{ }^{m} \mathcal{L}_{A^{\prime}} b_{<k}$. Since $\left.c^{m}\right\rfloor_{A^{\prime}} b_{\leq k}$, we get $b_{k}{ }^{m} \Psi_{A^{\prime}} c b_{<k}$. By regularity of $o\left(b_{k} / A^{\prime}\right)=o\left(a^{\prime} / A^{\prime}\right)$ we obtain $b_{k}{ }^{m} \mathcal{L}_{A^{\prime} c b_{<k}} a^{\prime}$; this yields $b_{k}{ }^{m} \mathcal{L}_{A^{\prime}} a^{\prime} c b_{<k}$, a contradiction.

We define the notions of domination exactly as in [17, Definition 5.2.6]. $a$ and $b$ below are elements (or finite tuples) and $C$ is a finite set containing sets $A$ and $B$.

Definition 3.23 1. We say that a dominates $b$ over $A$, written $a>_{A} b$, if $\left.b^{m}\right\rfloor_{A} c$ for all $c \downarrow^{m}{ }_{A} a$. $a$ and $b$ are equidominant over $A$, denoted $a \doteq_{A} b$, if $a>_{A} b$ and $b>_{A} a$.

2. We say that $o(a / A)$ is more dominant than $o(b / B)$ over $C$, in symbols $o(a / A)>_{C}$ $o(b / B)$, if there are $a^{\prime} \in o(a / A)$ and $b^{\prime} \in o(b / B)$ such that $a^{\prime^{m}} \downarrow_{A} C, b^{\prime m} \downarrow_{B} C$ and $a^{\prime} \gg_{C} b^{\prime} . o(a / A)$ is more dominant than $o(b / B)$, written $o(a / A) \gg_{0} o(b / B)$, means that $o(a / A)>_{C} o(b / B)$ for some $C$.

3. We say that $o(a / A)$ is equidominant with $o(b / B)$ over $C$, in symbols o $(a / A) \doteq_{C}$ $o(b / B)$, if there are $a^{\prime} \in o(a / A)$ and $b^{\prime} \in o(b / B)$ such that $a^{\prime^{m}} \downarrow_{A} C, b^{\prime m} \mathcal{L}_{B} C$ and $a^{\prime} \doteq_{C} b^{\prime}$. Finally, $o(a / A)$ and $o(b / B)$ are equidominant, written $o(a / A) \dot{=}_{0} o(b / B)$, if $o(a / A) \doteq_{C} o(b / B)$ for some $C$.

Equidominance over a fixed set is an equivalence relation on elements, whereas it may not be an equivalence relation on orbits (it is easy to find an example of an orbit with two $m$-independent extensions which are $m$-orthogonal).

We have all the basic properties of domination, e.g.: if $w\left(o_{1}\right)=1$ and $o_{1}$ is not $m$-orthogonal to $o_{2}$ over a set $A$ (containing the domains of $o_{1}$ and $o_{2}$ ), then $o_{2} \gg_{A} o_{1}$. If additionally $w\left(o_{2}\right)=1$, we get $o_{2} \doteq_{A} o_{1}$. In particular, by Proposition 3.22, if a regular orbit is not $m$-orthogonal to an orbit of weight 1 over $A$, then these orbits are equidominant over $A$ (which together with Proposition 3.19 is used in the proof of the next theorem).

Having all these results, we can repeat the proof of Theorem 5.2.18 of [17] to get the main theorem of this section.

Theorem 3.24 Each orbit in an m-stable compact ei-structure is equidominant with a product of finitely many regular orbits (hence of weight 1 ).

\section{References}

[1] E. Casanovas, D. Lascar, A. Pillay, M. Ziegler. Galois groups of first order theories. J. Math. Logic., 1:305-319, 2001.

[2] E. Hrushovski. Simplicity and the Lascar group. Preprint 1997.

[3] B. Kim. A note on Lascar strong types in simple theories. J. Symb. Logic., 63:926-936, 2000. 
[4] K. Krupiński. Products of finite abelian groups as profinite groups. J. Alg., 288:556-582, 2005.

[5] K. Krupiński. Abelian profinite groups. Fund. Math., 185:41-59, 2005.

[6] K. Krupiński. Profinite structures interprtable in fields. Ann. Pure Appl. Logic, to appear.

[7] K. Krupiński. Polish structures. In preparation.

[8] K. Krupiński, L. Newelski. On bounded type-definable equivalence relations. Notre Dame J. Formal Logic, 43:231-242, 2002.

[9] K. Krupiński, F. Wagner. Small profinite groups and rings. J. Alg., to appear.

[10] D. Lascar, A. Pillay. Hyperimaginaries and automorphism groups. J. Symb. Logic, 66:127-143, 2001.

[11] L. Newelski. $\mathcal{M}$-gap conjecture and m-normal theories. Israel J. Math., 106:285311, 1998.

[12] L. Newelski. Flat Morley sequences. J. Symb. Logic, 64:1261-1279, 1999.

[13] L. Newelski. Geometry of *-finite types. J. Symb. Logic, 64:1375-1395, 1999.

[14] L. Newelski. Small profinite groups. J. Symb. Logic, 66:859-872, 2001.

[15] L. Newelski. m-normal theories. Fund. Math., 170:141-163, 2001.

[16] L. Newelski. Small profinite structures. Trans. Amer. Math. Soc., 354:925-943, 2002.

[17] F. O. Wagner. Simple Theories. Kluwer Academic Publishers, Dordrecht, The Netherlands, 2000.

[18] F. O. Wagner. Small profinite m-stable groups. Fund. Math., 176:181-191, 2003.

Mathematical Institute, University of Wrocław

pl. Grunwaldzki 2/4, 50-384 Wrocław, Poland.

e-mail: kkrup@math.uni.wroc.pl

and

Mathematics Department

University of Illinois at Urbana-Champaign

1409 W. Green Street, Urbana, IL 61801, USA. 